\title{
Una mirada a la educación para el desarrollo sostenible y el ambiente en las universidades públicas de Honduras
}

\begin{abstract}
A look at education for sustainable development and the environment in the public universities of Honduras
\end{abstract}

Tania Teresa Najarro Vargas*

najarro.tania@gmail.com

Carlos Roney Montenegro Mejía**

cmontenegro@upnfm.edu.hn

\section{Resumen}

Repensar los valores de la sociedad humana para reconocer la «Tierra Patria» que propone Edgard Morín, que en el lenguaje de las Naciones Unidas se denomina desarrollo sostenible, marca una tendencia hacia las políticas de educación superior, el comportamiento de la generación de conocimiento e información a través de publicaciones y las declaraciones de políticas de las universidades públicas es de utilidad para identificar las acciones nacionales

\footnotetext{
* Estudiante del Doctorado Latinoamericano en Políticas Públicas y Formación Docente, UPNFM, Honduras.

** Estudiante del Doctorado Latinoamericano en Políticas Públicas y Formación Docente, UPNFM, Honduras.

Recibido 31 de agosto de 2018 / Aceptado 28 de septiembre de 2018

DOI: https://dx.doi.org/10.5377/paradigma.v25i40.7547

$<$ Paradigma> Revista de Investigación Educativa 40

Noviembre 2018/ ISSN: 1817-4221

Los articulos de la Revista Electrónica Paradigma del Instituto de Investigación y Evaluación Educativas y Sociales de la Universidad Pedagógica Nacional Francisco Morazán, de Honduras, se comparten bajo térrminos de la Licencia Creative Commons: Se permite que otros puedan descargar las cbras y compartirlas con otras personas, siempre y cuando se reconozca su autoría, pero no se pueden cambiar de ninguna manera ni se pueden utilizar comercialmente.
} 
hacia esta tendencia. En esta investigación se identificó el interés en publicaciones de artículos indexados y publicaciones institucionales en el tema. En Honduras los documentos de modelos educativos incluyen, a nivel de macroestructura y mesoestructura, elementos que muestran intentos de integrar dimensiones del desarrollo sostenible como la dimensión social y ambiental. Los esfuerzos nacionales están siendo completados con apoyo de movimientos en la región centroamericana, como es el caso de la transversalización del currículo con los temas cambio climático y gestión de riesgo.

Palabras clave: educación superior, educación ambiental, desarrollo sostenible, publicaciones, universidades públicas

\section{Abstracts}

It's necessary to rethink the values of the human society to recognize the "Homeland" proposed by Edgar Morin, called sustainable development, by the language of the United Nations. This marks a trend toward policies of higher education, the behavior of the generation of knowledge and information through publications and policy statements of public universities is useful to identify national actions for this trend. In this investigation interest was identified in publications of indexed articles and institutional publication on the subject. In Honduras, educational model documents includes at levels of macrostructure and mesostructure elements that show attempts to integrate dimensions of sustainable development such as social and environmental dimension. The national efforts are being completed with the support of movements in the Central American region, such as the mainstreaming of the curriculum with the themes of climate change and risk management. 
Keywords: higher education, educational politics, sustainable development, publications, public universities

\section{Introducción}

La necesidad de repensar en los valores de la sociedad humana ha venido tomando relevancia debido a la historia de destrucción que han generado los diferentes abordajes económicos de todas las civilizaciones con el fin de suplir sus necesidades, generando un desequilibrio ecológico, el cual se ha venido visibilizando por pensadores, académicos y científicos, cuya producción ha provisto de una visión que ha marcado la ruta para la toma de decisiones razonada, que paulatinamente, ha venido influyendo el comportamiento de las sociedades y los individuos, en sus esfuerzos por generar un desarrollo respetuoso del ambiente, que permita satisfacer de manera más integral y a través de varias generaciones, esas necesidades para tener una vida plena y en libertad (Sen, 2000 pp. 6-12).

Morín (2006, p. 35), plantea que en el siglo XX, la economía, la demografía, el desarrollo y la ecología se han transformado en un problema, vinculado a todas las naciones y civilizaciones, es decir al planeta en su conjunto, para lo que se debe generar una conciencia de «Tierra Patria», este y otros análisis al respecto han generado una tendencia de las políticas globales que han influenciado a las políticas educativas, siendo esta última una herramienta promovida por organismos como la UNESCO, para alcanzar estas libertades en el desarrollo, como lo plantea Amartya Sen en su premiada obra «Libertades y Desarrollo» de 1999.

Los articulos de la Revista Electrónica Paradigma del Instituto de Investigación y Evaluación Educativas y Sociales de la Universidad Pedagógica Nacional Francisco Morazán, de Honduras, se comparten bajo términos de la Licencia Creative Commons: Se permite que otras puedan descargar las obras y compartirlas con otras personas, siempre y cuando se reconozca su autoría, pero no se pueden cambiar de ninguna manera ni se pueden utilizar comercialmente. 
Este artículo tiene como propósito analizar cómo se han comportado las publicaciones sobre la temática de educación para el desarrollo sostenible y su pilar: la educación ambiental, en las regiones y países cercanos a Honduras, y como estas tendencias de políticas para educación superior han sido abordadas en universidades públicas de Honduras.

\section{Metodología}

En este artículo se da respuesta a dos preguntas de investigación: ¿cómo se han comportado las publicaciones sobre la temática de educación para el desarrollo sostenible y su pilar, la educación ambiental, en las regiones y países cercanos a Honduras? y ¿cómo estas tendencias de políticas para educación superior han sido abordadas en universidades públicas de Honduras? Para esto se desarrolló una metodología en dos fases:

La primera fase que hace énfasis en una lectura sobre cómo se ha comportado la producción de publicaciones para la integración de la temática de desarrollo sostenible en las políticas de educación superior, como una forma de racionalización de lo que debería ser la política educativa de educación superior.

Se realizó una búsqueda de publicaciones con lineamientos o análisis que favorecieran la inclusión de la educación para el desarrollo sostenible en las políticas educativas de educación superior; se tomaron en cuenta las publicaciones en el periodo del 2005 al 2017, con la utilización de criterios de elegibilidad de los artículos a considerarse, que hacen mención a América Latina, Centroamérica y particularmente a Honduras. Haciendo uso de las palabras claves en inglés y en español, establecidas previamente: educación superior, políticas educativas, desarrollo local, desarrollo humano, ambiente, 
desarrollo sostenible, se utilizaron 7 buscadores académicos especializados: Scielo, Redalyc, Google académico, Dialnet, Spoct host, Clacso y Repositorios universitarios nacionales, encontrándose un total de 70 publicaciones con las características establecidas; $43 \%$ de las cuales fueron de revistas indexadas y el $57 \%$ publicaciones institucionales.

En la segunda fase, se hace un análisis de las políticas de hecho de universidades públicas de Honduras, a través de una lectura de contenido, observando aspectos relevantes como ser: declaración de inclusión en el modelo educativo, carreras específicas y su objetivo de incorporación, así como clases o espacios pedagógicos específicos y sus objetivos de incorporación, identificando también el abordaje específico de problemas ambientales globales, como la gestión del riesgo y el cambio climático, análisis que nos permite ver cómo Honduras está integrando la educación para el desarrollo sostenible en las universidades públicas. El análisis de ambas fases permite llegar a algunas consideraciones generales que aportan a esta labor de creciente interés.

\section{La educación ambiental y el desarrollo sostenible como una tendencia en las políticas educativas}

Para la UNESCO, la educación se ocupa de la promoción de valores democráticos, la convivencia civilizada y la actividad autónoma y responsable de las personas, todo esto ligado con las libertades promovidas como parte esencial del desarrollo. En la teorización de Sen (2000, p. 16) «el desarrollo consiste en la eliminación de algunos tipos de faltas de libertades que dejan a los individuos pocas opciones y escasas oportunidades para ejercer esa agencia razonada»; hace de la educación para el desarrollo sostenible un abordaje razonado que vislumbra un particular esfuerzo para las nuevas generaciones 
de políticas, particularmente para las políticas educativas, la que se constituye como una tendencia desde finales del siglo $X X$.

Fuentes y Gonzales (2016, pp. 222-223), mencionan que desde 1972, con la conferencia de Estocolmo, se logró vincular la problemática ambiental a la esfera de lo social y cultural. En 1977, se realizó la primera Conferencia Internacional sobre Educación Ambiental en Tbilisi, donde se evidenció la necesidad de incluir la educación ambiental en la formación de todos los individuos y las sociedades. En 1981 se creó la Red de Formación Ambiental para Latinoamérica, con el auspicio del PNUMA, para propiciar alternativas de formación en la región.

Para la década de los 80, ya participan en la gestión ambiental sectores económicos y políticos. Con una visible manifestación global de la contaminación, disminución de la capa de ozono, el efecto invernadero y el consecuente cambio climático, se realiza el Informe titulado «Nuestro futuro común» de 1987, conocido también como «Informe Brundtland».

Es hasta el año 1992, durante la cumbre de Río de Janeiro que se adoptaron como ejes del desarrollo de la sensibilización, de la formación y educación relativas al ambiente y se reconoció que la problemática ambiental se relacionaba con el desarrollo sostenible (Fuentes y Gonzales, 2016, p.13).

En esa misma época pensadores de la educación como Paulo Freire (1995) y Gadotti (1997) explican la ecopedagogía como movimiento social y político complejo, que supone la integración de aspectos políticos, económicos, culturales, históricos y aspectos de cambio general en los procesos de reestructuración curricular (Fuentes y Gonzales, 2016, p. 219). 
La temática del desarrollo sostenible va generando espacios especializados para su abordaje. En el 2000 la Asamblea General de la Organización de las Naciones Unidas proclamo «el Decenio para Educación para el Desarrollo Sostenible» y proclamó a la UNESCO como organismo rector de la promoción y aplicación del decenio (UNESCO, 2005b. p. 1). Para este fin, la UNESCO declara «Necesitamos una educación distinta, una educación que pueda contribuir en la formación de ciudadanas y ciudadanos que sean capaces de construir un futuro posible y sostenible, lo que se ha llamado educación para el desarrollo sostenible» e instaura la década de la «Educación para el Desarrollo Sostenible (2005-2014)» (UNESCO, 2005a, p. 2). Esta iniciativa se complementa y potencia con otras iniciativas, como los objetivos del milenio, en una demostración global de preocupación por el futuro.

El planteamiento de la UNESCO es que la educación es el factor determinante de la transición al desarrollo sostenible; parafraseando a Morín (2006, p. 1), generar en los países la conciencia planetaria, lo que requerirá un cambio en la concepción de este «desarrollo» que debe pasar por el reconocimiento de la «Tierra Patria». La comunidad internacional entonces propone una serie de adecuaciones en los Estados y en la cooperación que permitan potenciar esta propuesta de un futuro global «Esto nos exige una nueva orientación de los sistemas, como de las políticas, contenidos y prácticas, con el fin que todos y cada uno de nosotros podamos tomar decisiones y actuar de manera apropiada y pertinente...» (UNESCO, 2005, p. 3).

Según Beech y Meo (2016, p. 5), en estudios sobre las herramientas propuestas por Ball se destaca la importancia de entender las políticas educativas más allá del estado-nación, además plantea la necesidad de reconocer:

Los procesos de gobernanza y las heterarquías como formas alternativas de ejercer el poder constituyen y son constituidos por

Los articulos de la Revista Electrónica Paradigma del Instituto de Investigación y Evaluación Educativas y Sociales de la Universidad Pedagógica Nacional Francisco Morazán, de Honduras, se comparten bajo térrminos de la Licencia Creative Commons: Se permite que otras puedan descargar las obras y compartirlas con otras personas, siempre y cuando se reconozca su autoría, pero no se pueden cambiar de ninguna manera ni se pueden utilizar comercialmente. 
nuevos espacios de política. La política educativa está siendo creada por nuevos actores que establecen nuevas fuentes de autoridad. Se trata de una arquitectura global de relaciones políticas que incluye a los estados nacionales y subnacionales, organizaciones internacionales (como la OCDE, UNESCO y el Banco Mundial), empresas multinacionales, organizaciones de la sociedad civil, consultoras y otras organizaciones hibridas, como las empresas que usan mecanismos de mercado para abordar problemas sociales o ambientales (Beech y Meo, 2016, p. 7).

\section{Producción de publicaciones sobre desarrollo sostenible en las políticas de educación superior}

La producción en publicaciones es de suma importancia. Estudios realizados por la UNESCO (2016, p.13) muestran como «los tomadores de decisiones tienen, por lo general, grandes dificultades para comprender y responder de manera efectiva a los problemas complejos cuando el estado del conocimiento científico es incompleto y posiblemente divergente». Por lo que, para conocer el estado del conocimiento en la temática es relevante dar respuesta a la pregunta de investigación: ¿cómo se han comportado las publicaciones sobre la temática de educación para el desarrollo sostenible y su pilar la educación ambiental, en las regiones y países cercanos a Honduras? A continuación, se hace un recuento del estado de las publicaciones de la inclusión de la educación para el desarrollo sostenible y ambiente en las políticas educativas.

En general se puede decir que se ha generado información para la integración de la educación de desarrollo sostenible y educación ambiental, en la educación superior, tanto a nivel de científico a través publicaciones indexadas 
$42.86 \%$, como de las publicaciones institucionales, con el $57.14 \%$ del total de publicaciones identificadas (ver tabla 1).

Es importante notar como las publicaciones están concentradas haciendo referencias a América Latina con mayor porcentaje de publicaciones tanto indexadas como institucionales, esta referencia tiene la desventaja que, al estar la región constituida por países tan heterogéneos, se pierde la particularidad de cada uno. Para el ámbito centroamericano $7.14 \%$ es de las publicaciones institucionales y $2.86 \%$ de publicaciones indexadas. Cabe resaltar que Costa Rica tiene el mayor porcentaje (4.29\%) de publicaciones indexadas y Honduras es el país con el mayor porcentaje (12.86\%) de publicaciones institucionales.

Tabla 1.

Porcentajes por tipo de publicación (indexada o institucional) y regiones o países a los que hacen referencia en las publicaciones

\begin{tabular}{lcc}
\hline País/Región & $\begin{array}{l}\text { Porcentaje de } \\
\text { publicaciones } \\
\text { indexadas }\end{array}$ & $\begin{array}{c}\text { Porcentaje de } \\
\text { publicaciones } \\
\text { institucionales }\end{array}$ \\
\hline América Latina $^{\text {a }}$ & 31.43 & 35.71 \\
Centroamérica $^{\mathrm{b}}$ & 2.86 & 7.14 \\
Guatemala & 0.00 & 1.43 \\
El Salvador & 1.43 & 0.00 \\
Honduras & 1.43 & 12.86 \\
Nicaragua & 1.43 & 0.00 \\
Costa Rica & 4.29 & 0.00 \\
Total & 42.86 & 57.14 \\
\hline
\end{tabular}

$\mathrm{N}=70$

Nota: Elaboración propia a partir de búsqueda electrónica entre el 28 de arzo y el 19 de abril del 2017, con publicaciones de 1999 al 2017

Los articulos de la Revista Electrónica Paradigma del Instituto de Investigación y Evaluación Educativas y Sociales de la Universidad Pedagógica Nacional Francisco Morazán, de Honduras, se comparten bajo térrminos de la Licencia Creative Commons: Se permite que otras puedan descargar las obras y compartirlas con otras personas, siempre y cuando se reconozca su autoría, pero no se pueden cambiar de ninguna manera ni se pueden utilizar comercialmente. 
a publicaciones que refieren al conjunto de los países de América latina ${ }^{\mathrm{b}}$ publicaciones que refieren al conjunto de los países de Centroamérica

Las referencias específicas de las publicaciones a las realidades de cada uno de los países es relativamente poca, se puede observar que la mirada regional, ha sido generada en espacios comunes del conocimiento, como ser el caso de la Red de Universidades Iberoamericanas (UNIVERSIA) y en el caso de Centroamérica su Consejo Superior Universitario Centroamericano (CSUCA), que generó líneas de acción explícitas para la integración de la temática en los países a través del Tercer Plan para la Integración Regional de la Educación Superior para Centroamérica (CSUCA, 2013, p. 17).

Esta generación de información se vio marcada cuando la UNESCO instaura la década de la «Educación para el Desarrollo Sostenible (2005-2014)» (UNESCO, 2005a, p. 2) ya que analizando publicaciones desde el año 1999 a la fecha (ver gráfica 1) se observa un incremento del número de publicaciones durante el decenio.

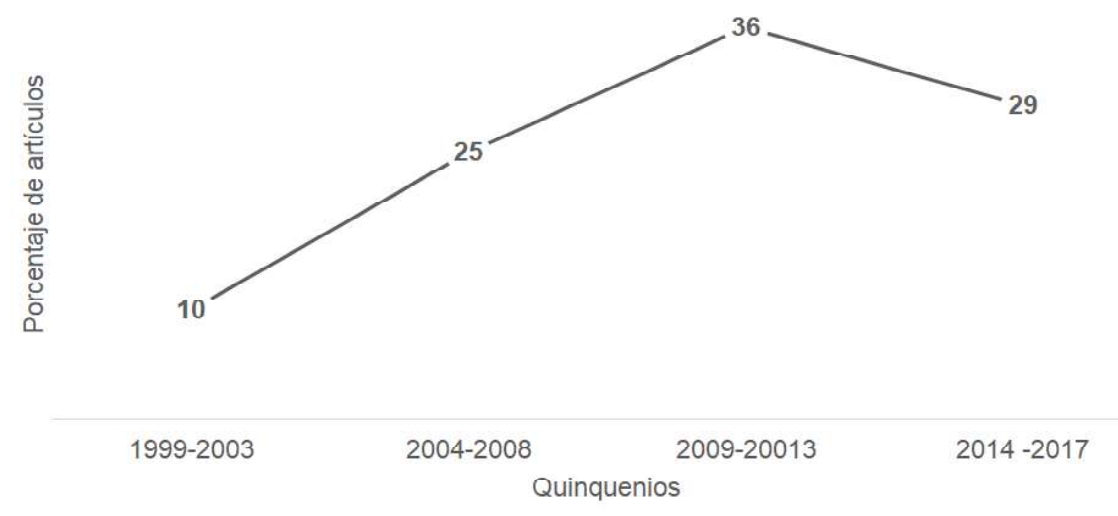

Figura 1. Grafica de porcentaje de publicaciones en cuatro quinquenios, por Najarro y Montenegro, a partir de búsqueda electrónica entre el 28 de marzo y 19 de abril del 2017, con publicaciones de 1999 al 2017. 
El número de publicaciones es considerado como un indicador de actividad científica (UN, 2007, p. 7), por lo que es interesante encontrar que para América Latina y Centroamérica durante el 2004 y el 2013, hubo un incremento en las publicaciones, confirmando lo observado por otros autores como Sepúlveda (2015, p. 5) que en su análisis mundial, sobre publicaciones del tema, señala «Ya en torno a la producción puntual de artículos en el periodo 2005-2014, se puede apreciar una tendencia creciente...con una notable disminución en los años 2011 y 2012».

En base a las publicaciones revisadas los temas abordados se muestran en la gráfica a continuación:

El número de publicaciones es considerado como un indicador de actividad científica (UN, 2007, p. 7), por lo que es interesante encontrar que para América Latina y Centroamérica durante el 2004 y el 2013, hubo un incremento en las publicaciones, confirmando lo observado por otros autores como Sepúlveda (2015, p.5) que en su análisis mundial, sobre publicaciones del tema, señala «Ya en torno a la producción puntual de artículos en el periodo 2005-2014, se puede apreciar una tendencia creciente ...con una notable disminución en los años 2011 y 2012».

En base a las publicaciones revisadas los temas abordados se muestran en la gráfica a continuación: 


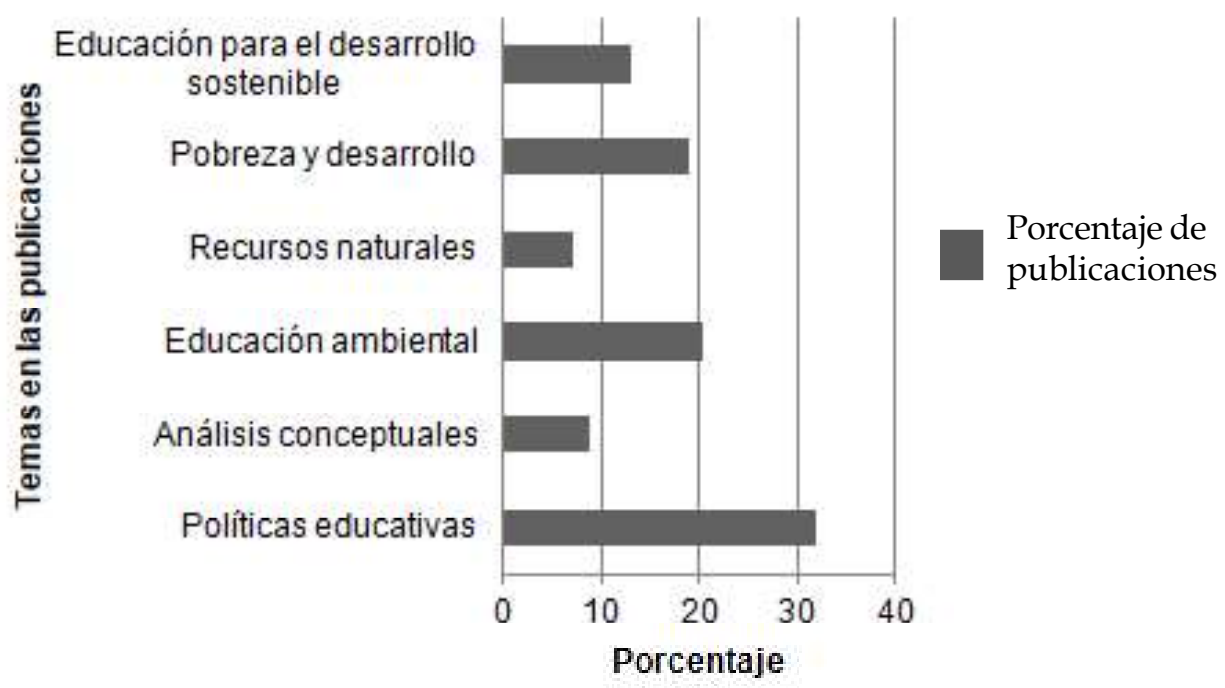

Figura 2. Grafica de porcentaje de temas abordados en el total de las publicaciones analizadas, por Najarro y Montenegro, a partir de búsqueda electrónica entre el 28 de marzo y el 19 de abril del 2017, con publicaciones del 1999 al 2017

El porcentaje más alto de temas abordados fue el de las políticas educativas en más del 30\% de las publicaciones, las cuales están en su mayoría contextualizadas de manera genérica a América Latina, con algunas menciones particulares para Centro América, se recuperaron casos para El Salvador, Honduras y Nicaragua. Álvarez et al (2014, p. 35) discuten la dinámica de la formulación de las políticas educativas en la región y aseveran que las mismas son «más orientadas por prioridades establecidas en cumbres internacionales o en planes impulsados por organismos y agencias internacionales, que por prioridades nacionales derivadas de diagnósticoso estudios independientes de los países», dando así relevancia a la tendencia de políticas promovidas a nivel internacional.

En el tema, educación ambiental, que fue discutido por el $20 \%$ de las publicaciones, Medina y Paramo (2014, p. 55) mencionan que a pesar del 
gran auge que ha tenido el desarrollo de programas y experiencias en educación ambiental, en la región latinoamericana, son pocos los artículos que exploran de manera sistemática su efectividad en la formación de las personas. Bravo (2012, p. 3), describe como la educación ambiental aparece en la década de los setenta: surge en el marco de la Conferencia de las Naciones Unidas sobre el Medio Humano realizada en Estocolmo (1972), sin embargo, para 1995 la UNESCO cancela el Programa Internacional de Educación Ambiental y pretende sustituirla por la Educación para un futuro sustentable, propuesta planteada en la Conferencia Mundial de Medio Ambiente y Sociedad: Educación y Sensibilización para la Sostenibilidad en 1997, por lo que la educación ambiental es una de las formas de implementación de la educación para el desarrollo sostenible con más tradición de abordaje teórico y práctico, sin embargo es una visión parcial que debe ser ampliada frente a los actuales problemas globales, que están vinculados a sistemas económicos y distribución de riquezas.

\section{Honduras y la integración del tema desarrollo sostenible en las universidades públicas}

Honduras no está desvinculada de las tendencias internacionales relativas al desarrollo sostenible y el ambiente en la educación superior. Para dar respuesta a la pregunta: ¿cómo estas tendencias de políticas para educación superior han sido abordadas en universidades públicas de Honduras? se analizaron documentos oficiales, debido a que la integración de temáticas especificas responde al abordaje que se den de estos temas en las políticas o declaraciones.

a través del análisis de texto recomendadas por Ball en Beech y Meo (2016, p. 54). 
Esa integración en el texto se vuelve particularmente significativa si se trata del modelo educativo, ya que según Tunnermann (2008 p. 15):

El modelo educativo es la concreción, en términos pedagógicos, de los paradigmas educativos que una institución profesa y que sirve de referencia para todas las funciones que cumple (docencia, investigación, extensión, vinculación y servicios), a fin de hacer realidad su proyecto educativo.

Por lo anterior la inclusión de las tres dimensiones del desarrollo sostenible: económica, ambiental y social, en el modelo educativo es de suma relevancia. En ese sentido la tabla 2 muestra como las dos universidades públicas en Honduras han integrado en su modelo educativo principalmente el principio de equidad, dando relevancia a la dimensión social del desarrollo sostenible.

Tabla 2.

Aspectos del modelo educativo de la UNAH y la UNPFM (como integran en su texto el desarrollo sostenible)

\begin{tabular}{|c|c|c|}
\hline $\begin{array}{l}\text { Aspecto del modelo } \\
\text { educativo }\end{array}$ & UNAH & UNPFM \\
\hline Principios & $\begin{array}{l}\text { Hace mención a } \\
\text { la equidad }\end{array}$ & $\begin{array}{l}\text { Hace mención a } \\
\text { la equidad }\end{array}$ \\
\hline $\begin{array}{l}\text { Perspectiva } \\
\text { Pedagógica }\end{array}$ & $\begin{array}{l}\text { Teoría constructivista, fun- } \\
\text { damentada en teorías } \\
\text { cognitivas y de aprendiza- } \\
\text { je, la teoría crítico-reflexiva } \\
\text { y humanista }\end{array}$ & $\begin{array}{l}\text { Es la psicología educativa co- } \\
\text { existen diversos paradigmas } \\
\text { conductista, humanista, cogni- } \\
\text { tivo, psicogenético y socio-cul- } \\
\text { tural, constructivista, se } \\
\text { visualizan diferentes tenden- } \\
\text { cias de la investigación } \\
\text { psicológica y educativa. }\end{array}$ \\
\hline
\end{tabular}

Fuente: Elaboración propia, en base a Modelo educativo de la UNAH (2009) y Modelo educativo de la UPNFM (2014) 
La importancia de la perspectiva pedagógica se encuentra en que es el fundamento para la construcción de diseños curriculares. En ambas universidades se consideraron perspectivas que permiten la inclusión activa de las tres dimensiones del desarrollo sostenible, al menos de manera intencional, currículos de la UNAH y la UPNFM. Estas universidades, con mayor población estudiantil en el país, consideran la dimensión ambiental de manera obligatoria para sus estudiantes y el principio de equidad de la dimensión social, como se muestra en la tabla 3:

Tabla 3

Clases o espacios pedagógicos específicos de las universidades públicas de Honduras y el objetivo de incorporación frente a la educación ambiental y el desarrollo sostenible

$\begin{array}{lll}\text { Universidad } & \begin{array}{l}\text { Clase/espacio } \\ \text { pedagógico específico }\end{array} & \begin{array}{l}\text { Objetivo de } \\ \text { Incorporación }\end{array}\end{array}$

UNAH La clase de educación El estudiante valorarála importancia ambiental (obligatoria como de preservar el equilibrio del sistema parte de las clases generales ambiental para garantizar la sobrepara todas las carreras) vivencia de la humanidad.

UPNFM La Cátedra de la Tierra «Dr. Gonzalo Cruz Calderón», (requisito de graduación)

Es una estrategia educativa que incluye una amplia divulgación del conocimiento científico y la realización de acciones prácticas para fortalecer una conciencia proactiva hacia el equilibrio del planeta tierra, a través de los

Ciencias de la tierra

Pretende que el estudiante adquiera los elementos esenciales para entender la estructura y dinámica del planeta tierra y semejanzas y diferencias con otros planetas del sistema solar.

Los articulos de la Revista Electrónica Paradigma del Instituto de Investigación y Evaluación Educativas y Sociales de la Universidad Pedagógica Nacional Francisco Morazán, de Honduras, se comparten bajo términos de la Licencia Creative Commons: Se permite que otros puedan descargar las obras y compartirlas con otras personas, siempre y cuando se reconozca su autoría, pero no se pueden cambiar de ninguna manera ni se pueden utilizar comercialmente. 
Comprende tópicos fundamentales en geología y geofísica, meteorología, oceanografía y física planetaria, así como sus influencias en las condiciones ambientales, los ecosistemas, las fuentes energéticas y las consecuencias en la calidad de vida en el planeta.

Introducción a la Educación

Ambiental
Tiene como propósito presentar una visión global de los elementos que forman parte del medio natural, así como el análisis de las relaciones entre la población humana y el medio ambiente, a fin de fomentar una actitud crítica y de compromiso en el mejoramiento y conservación de este.

Nota: Elaboración propia, en base al Documento de creación de carreras UNA (2006). Informe temático de desarrollo sostenible en las funciones de UNACIFOR y sus ofertas académicas.

La UNAH y la UPNFM cuentan con clases o espacios pedagógicos obligatorios para los estudiantes de todas las carreras, constituyéndose este como un paso hacia la ambientalización del currículo, uno de los desafíos de la ecopedagogía, que según Fuentes y Gonzales (2016, p. 221) debe estar articulada a las dinámicas académicas curriculares al mismo nivel de las otras prioridades de la formación universitaria, como espacio para promover la sensibilización y motivación hacia la concientización ambiental, mediante la gestión del conocimiento, con estrategias didácticas que generen un cambio de actitud y la participación por parte de los actores educativos.

Esfuerzos de transversalización de problemas ambientales complejos como lo es la gestión del riesgo y cambio climático han sido dirigidos por la tendencia en la región centroamericana a través del CSUCA. Este interés por abordar temáticas ambientales globales, como se mencionó anteriormente, es manifiesto por el CSUCA, a través de la Política Universitaria 
Centroamericana para la Reducción del Riesgo de Desastres (PUCARDD), en su edición de febrero del 2017, a través de la cual se adopta un marco conceptual y regulatorio para la reducción de riesgo de desastres para Universidades Centroamericanas. En él se identifica la contribución de las universidades a esta problemática y se definen componentes que abarcan temas como ser: la gestión institucional Universitaria, lo académico, la seguridad interna y el compromiso con la comunidad y el país (pp. 11 - 38).

Esta declaración de política centroamericana ha generado proyectos con cuyo financiamiento se han implementado iniciativas en diferentes universidades del país, tanto públicas como privadas, que han relacionado su análisis de desastres a cambio climático, como se mostró en el taller nacional de trasferencia del conocimiento y divulgación de resultados en reducción de riego a desastres (RRD), realizado el 5 de marzo del 2018 en la ciudad de Tegucigalpa, en donde representantes de las universidades que han sido parte de estas iniciativas participaron en el panel: Experiencias universitarias en gestión de riesgo y adaptación al cambio climático que operativizan la PUCARDD. En el cuadro a continuación se muestra la iniciativa realizada por universidades de Honduras participantes:

Iniciativas de gestión de riesgo en las Universidades de Honduras en el marco del apoyo regional 2018. 
Tabla 4

Iniciativa de gestión de riesgo en universidades de Honduras

\begin{tabular}{ll}
\hline Universidad & Iniciativa \\
\hline Escuela Agrícola & «Fortalecimiento de la Gestión Ambiental en \\
Panamericana, & Adaptación al Cambio Climático (ACC) y \\
Zamorano & $\begin{array}{l}\text { Reducción del Riesgo a Desastres (RRD) en } \\
\text { Zamorano» }\end{array}$
\end{tabular}

Universidad Nacional de Agricultura

Fortalecimiento de la gestión de riesgos y adaptación al cambio climático en la currícula educativa de cinco carreras en la Universidad Nacional de Agricultura.

Universidad Nacional de Ciencias Forestales

Participa como miembro en la red universitaria de Latino América y El Caribe para la reducción de riesgos a desastres (REDULAC-RRD).

Universidad Nacional Universidad Segura, Elaboración e impleAutónoma de Honduras mentación de un plan de gestión de riesgo usando enfoque de RDD, basado en la comunidad.

Inserción del eje transversal de gestión de riesgo y cambio climático en 6 carreras del CURLA-UNAH

Universidad Pedagógica Francisco Morazán

Se desarrolló el proyecto de Gestión Integral de Riesgo de desastres y Adaptación al Cambio Climático en los planes de Estudios en 80 espacios pedagógicos de la Facultad de Humanidades y en 60 espacios pedagógicos de la Facultad de Ciencia y Tecnología, con el auspicio, en el CUED, San Pedro Sula y La Ceiba. CSUCA-PRIDCA/ COSUDE. (2014- 2015)

Fuente: elaboración propia a partir de las presentaciones del Panel: Experiencias universitarias en gestión de riesgo y adaptación al cambio climático que operativizan la PUCARDD, 2018. 
Todo esto ha generado una experiencia en el abordaje de temáticas de la agenda nacional e internacional, este valioso esfuerzo puede ser completado con análisis ex post que permitan conocer el impacto de éste, además sirve como base para generar información ampliada al cambio climático, como fenómeno que impacta los medios de vida locales más allá de los fenómenos climáticos extremos que generan desastres.

La incorporación del desarrollo sostenible y ambiente a nivel de texto se ha visto en los modelos educativos, hay otros niveles de concreción de los modelos educativos que deben analizarse para dar cuentas de abordaje integral como ser el nivel de mesoestructura, a través de unidades académicas y carreras. En la tabla 5, podemos observar carreras con la intencionalidad explicita en el tópico; en este análisis integramos dos universidades más como ser la Universidad Nacional de Ciencias Forestales (UNACIFOR) y la Universidad Nacional de Agricultura (UNA) ya que ambas han sido las encargadas directamente de carreras con relación directa en el manejo de los recursos naturales; en la UNAH y en la UNACIFOR el objetivo de incorporación está alineado en su totalidad con el desarrollo sostenible.

Tabla 5.

Carreras específicas de las universidades públicas de Honduras y el objetivo de incorporación frente a la educación para el desarrollo sostenible y ambiente

\begin{tabular}{lll}
\hline Universidad & Carreras & Objetivo de incorporación \\
\hline UNACIFOR & $\begin{array}{l}\text { Técnico } \\
\text { universitario en } \\
\text { dasonomía }\end{array}$ & $\begin{array}{l}\text { Es importante el manejo de los recursos } \\
\text { naturales de forma sustentable, en } \\
\text { especial el suelo, el agua, el aire, ya que } \\
\text { de ellos dependen los demás recursos; a }\end{array}$ \\
& $\begin{array}{l}\text { Ingeniería en } \\
\text { c i e n c i a s se requiere satisfacer las } \\
\text { forestales }\end{array}$ & $\begin{array}{l}\text { necesidades de una sociedad cada vez } \\
\text { más exigente; situación que en el país se } \\
\text { ha agudizado por los efectos de la }\end{array}$
\end{tabular}

Los articulos de la Revista Electrónica Paradigma del Instituto de Investigación y Evaluación Educativas y Sociales de la Universidad Pedagógica Nacional Francisco Morazán, de Honduras, se comparten bajo términos de la Licencia Creative Commons: Se permite que otros puedan descargar las obras y compartirlas con otras personas, siempre y cuando se reconozca su autoría, pero no se pueden cambiar de ninguna manera ni se pueden utilizar comercialmente. 
Ingeniería en e $\mathrm{n}$ e $\mathrm{r} g$ í a renovable

UNA

\begin{abstract}
Carrerade recursos naturales y ambiente
\end{abstract}

En el grado de licenciatura Código: C2-RNA deforestación, contaminación hídrica y en general ambiental por el mal manejo de los recursos naturales. Adicionalmente el aporte del sector forestal al PIB tradicionalmente ha sido bajo y la balanza comercial deficitaria

Ante la enorme problemática ambiental -en general- y del aprovechamiento irracional de los recursos naturales -en particular- que se vive en Honduras, es indispensable y decisiva la formación de profesionales universitarios capaces de proponer y generar cambios que contribuyan con la erradicación de la pobreza y que conduzcan el país por la senda del complejo desarrollo equitativo y sostenible de los recursos naturales y del ambiente.

Fuente: Elaboración propia, según Modelo educativo (2017), hoja web UPNFM (2017), hoja web de la UNAH (2017)

\section{Consideraciones finales}

Las publicaciones sobre la temática de educación para el desarrollo sostenible y su pilar, la educación ambiental, en las regiones y países cercanos a Honduras, muestran un comportamiento creciente en el que se ha generado información para la integración de la educación de desarrollo sostenible y educación ambiental, en el nivel educación superior, tanto a nivel científico, a través publicaciones indexadas $42.86 \%$, como de las publicaciones institucionales con el $57.14 \%$ del total de publicaciones identificadas. A simismo, las publicaciones están concentradas haciendo referencias al conjunto de países de A mérica Latina con mayor porcentaje de publicaciones 
tanto indexadas como institucionales. Esta generación de información fue evidente cuando la UNESCO instauró la década para la educación del desarrollo sostenible (2004-2015).

Estas tendencias de políticas para educación superior han sido abordadas en universidades públicas en Honduras, mostrando como las dos universidades públicas han integrado en su modelo educativo principalmente el principio de equidad, dando relevancia a la dimensión social del desarrollo sostenible; así mismo, a nivel de currículo ambas universidades consideran la dimensión ambiental de manera obligatoria para sus estudiantes.

Quedan claros los esfuerzos de transversalización de los problemas ambientales globales como la gestión de riesgo y el cambio climático, por parte de la educación superior hondureña, a través del CSUCA, con quienes se han desarrollado proyectos específicos enmarcados en la Políticas Universitarias Centroamericanas para la Reducción del Riesgo de Desastres.

Las universidades públicas de Honduras han hecho significativos esfuerzos por integrar la educación ambiental y la educación para el desarrollo sostenible, a nivel de macro y meso estructura. Es importante mantener lo que Mainardes (2006, p. 54), resalta en el cuidado que según Ball (1993), se debe tener al analizar los textos de política «Mientras que la política como discurso hace hincapié en los límites impuestos por el propio discurso, la política como texto hace hincapié en el control que está en manos de los lectores», por lo que quedará pendiente conocer a profundidad como es el nivel de microestructura, docentes-estudiantes, para acercarnos más a la práctica de la educación para el desarrollo sostenible en las universidades de Honduras. 


\section{Recomendaciones}

En las universidades públicas de Honduras la visión debe ampliarse desde lo ambiental hacia la educación para el desarrollo sostenible, obligando así a una integralidad, no solo en las carreras relacionadas directamente con el manejo de recursos naturales, si no que optando por una abordaje más integral y multidisciplinario con relación a lo económico y lo social.

La necesidad de integralidad que requiere el esfuerzo de la educación para el desarrollo sostenible y educación ambiental, podría encontrar ideas y apoyo en los espacios regionales de análisis de potencialidades y como se observó en la lectura de producción de publicaciones indexadas e institucionales, los organismos internacionales pueden ofrecer alicientes para el incremento de la gestión del conocimiento, por lo que deberá haber una demanda, precisa desde los países y sus necesidades para incidir en estos apoyos, que sin duda serán constantes dado el interés en soluciones conjuntas de la problemática global, ambiental y social, de inequidad y economía frente a modelos que han mostrado ya su inviabilidad. Esta temática es vital para Honduras, pues el país actualmente ocupa uno de los primeros lugares de vulnerabilidad como el cambio climático a nivel mundial.

\section{Referencias}

Álvarez, A; Castillo, M; Elvir, A; Vijil, J. (2014). Quinto informe estado de la región. Las políticas de educación en Centroamérica. 144 p. Recuperado el 13 de abril de http://estadonacion.or.cr/files/ biblioteca_virtual/centroamerica/005/Educación/ LasPoliticasEducacionCA.pdf 
Beech, J. \& Meo, A. I. (2016). Explorando el uso de las herramientas teóricas de Stephen J. Ball en el estudio de las políticas educativas en América Latina. Archivos Analíticos de Políticas Educativas, 24(23). http:// dx.doi.org/10.14507/epaa.24.2417 Este artículo forma parte del número especial Stephen J. Ball y la investigación sobre políticas educativas en América Latina de EPAA/AAPE, Editores invitados: Jason Beech y Analía I. Meo.

Bravo, M. (2012). La UNAM y sus procesos de ambientalización curricular. Revista Mexicana de Investigación Educativa, octubre-diciembre, 11191146.

CSUCA. (2013). Tercer Plan para la Integración Regional de la Educación Superior Centroamericana. PIRESC III. Tercera Edición. Actualizado por el VII Congreso Universitario Centroamericano, realizado en León, Nicaragua, 14 y 15 de abril 2011. Ciudad Guatemala

Fuentes, N. \& Gonzales, H. (2016). Ambientalización del currículo universitario: un reto de la ecopedagogía. Tecné, Episteme y Didaxis: TED, (40), 310-339. Recuperado en agosto 2018 de: http:// education.esp.macam.ac.il/article/1737

Mainardes, J. (2006). Abordagem do ciclo de políticas: uma contribuição para a análise de políticas educacionais. Educ. Soc., Campinas, vol. 27, n. 94, p. 47-69, jan./abr. 200647 Disponível em http:// www.cedes.unicamp.br

Medina, I. \& Páramo, P. (2014). La investigación en educación ambiental en América Latina: un análisis bibliométrico. Revista Colombiana de Educación, (66), 55-72. 
Morín, E. (2006). Tierra Patria. Traducción Ricardo Figueria. Buenos Aires: Ediciones Buena visión

ONU. (1987). Report of the world commision on enviroment and development: Our common Future. Development and international economic co-operation: Environment. Assembly General A/42/427. Recuperado el 8 de abril del 2017 de: http://www.eng.mcmaster.ca/ sustainability/documents/OurCommonFuture.pdf

Sen, A. (2000). El desarrollo como libertad: Gaceta Ecológica. 14-20. Recuperado el 25 de abril de 2017, en http://www.redalyc.org/pdf/ 539/53905501.pdf

Sepúlveda, J. (2015). Estado de la investigación sobre educación para el desarrollo sostenible: un análisis cienciométrico de la producción científica en el período 2005-2014. Revista Luna Azul, JulioDiciembre, 309-322.

Tunnermann. C. (2008). Modelos educativos y académicos. Editorial Hispamer, Impreso en Nicaragua por Impresión Comercial La Prensa, S. A ISBN: 978-99924-79-35-3. Digitalizado por Enrique Bolaños. recuperado el 12 de octubre 2018 de: http:// www.enriquebolanos.org/media/publicacion/ Modelos\%20educativos\%20y\%20academicos.pdf

UNESCO. (2005). Macedo, B. Compromisos Educativos establecidos por la Década. Publ: 2005; 4 p. OREALC / 2005 / PI / H / 15. Recuperado el 8 de abril del 2017 de: http://unesdoc.unesco.org/images/0016/ 001621/162180s.pdf

UNESCO. (2016). Toma de decisiones y cambio climático: acercando la ciencia y la política en América Latina y el Caribe. Recuperado el 8 de abril del 2017 de: http://unesdoc.unesco.org/images/0024/ 002456/245647S.pdf 
UN. (2017). Thematic Think Piece: Science, technology and innovation for sustainable development in the global partnership for development beyond. ITU, OHCHR, UNCTAD, UNEP, UNESCO, UNFCCC, UNIDO, WIPO, WMO. Recuperada el 17 de abril 2017 http:// www.un.org/en/development/desa/policy/untaskteam_undf/ thinkpieces/28_thinkpiece_science.pdf

Universidad Nacional Autónoma de Honduras. (2009). Modelo Educativo de la UNAH: Tegucigalpa, Serie de publicaciones de la reforma universitaria No. 3 .

Universidad Pedagógica Nacional Francisco Morazán. (17 de Abril de 2017). Google Honduras. Obtenido de https://www.upnfm.edu.hn/

Universidad Pedagógica Nacional Francisco Morazán. (2011). Plan de estudios de la Carrera del Profesorado en la enseñanza del Turismo y la Hostelería en el grado de Licenciatura. Tegucigalpa: Rectoría, Vicerrectoría Académica, Facultad de Ciencia y Tecnología, Departamento de Ciencias Comerciales.

Universidad Pedagógica Nacional Francisco Morazán. (20014). Modelo Educativo de la Universidad Pedagógica Nacional Francisco Morazán. Tegucigalpa: VRAC-UPNFM.

Los articulos de la Revista Electrónica Paradigma del Instituto de Investigación y Evaluación Educativas y Sociales de la Universidad Pedagógica Nacional Francisco Morazán, de Honduras, se comparten bajo términos de la Licencia Creative Commons: Se permite que otros puedan descargar las obras y compartirlas con otras personas, siempre y cuando se reconozca su autoría, pero no se pueden cambiar de ninguna manera ni se pueden utilizar comercialmente. 
(C) $९$ Los articulos de la Revista Electrónica Paradigma del Instituto de Investigación y Evaluación Educativas y Sociales de la

BY NC ND Commons: Se permite que otros puedan descargar las obras y compartirlas con otras personas, siempre y cuando se
reconozca su autoria, pero no se pueden cambiar de ninguna manera ni se pueden utilizar comercialmente. 